\title{
Unabhängiges Landeszentrum für Datenschutz Schleswig-Holstein zur Stiftung Datenschutz: "So wird daraus nichts“
}

In ihrer Antwort auf die Kleine Anfrage des grünen Bundestagsabgeordneten Konstantin von Notz (BT-Drs. 17/8513) vom 13.02.2012 legt die Bundesregierung teilweise ihre Planungen offen zur „Stiftung Datenschutz“, „die Produkte und Dienstleistungen auf Datenschutzfreundlichkeit prüfen, Bildung im Bereich des Datenschutzes stärken, den Selbstdatenschutz durch Aufklärung verbessern und ein Datenschutzaudit entwickeln soll“: „Um das Stiftungsvermögen dauerhaft zu erhalten, sollen die Personalund Sachkosten gering gehalten werden. "Laufende, über den im Haushalt 2011 vorgesehene Zuwendungen in Höhe von 10 Mio. Euro sind nicht vorgesehen. Trotzdem ist als Gremium neben Vorstand und Verwaltungsrat ein 33köpfiger Beirat geplant, bei dem 15 der Mitglieder von der Wirtschaft gestellt werden sollen, um „Fachkompetenz und eine angemessene Interessenwahrnehmung durch unterschiedliche Beteiligte zu gewährleisten“. Die den Beiräten entstehenden Aufwendungen werden nicht erstattet. Der Satzungsentwurf der Stiftung sehe vor, „dass die Prüfung von Produkten und Dienstleistungen möglichst in Zusammenarbeit mit Stellen erfolgen soll, die sich die unabhängige Prüfung von Produkten und Dienstleistungen auf ihre Datenschutzfreundlichkeit zum Ziel gesetzt haben“.

Diese Planungen der Bundesregierung lassen nichts Gutes erhoffen. So wie sie dieses im Grunde richtige Projekt bisher angefasst hat, kann nichts daraus werden: Die Anregungen und Vorschläge der Datenschutzbehörden blieben bisher weitestgehend unberücksichtigt. Bei der Standortwahl werden ausschließlich Aspekte der Wirtschaftsförderung berücksichtigt, ohne an vorhandene Strukturen anzuknüpfen. Die Regierung meint anscheinend, Anreize zum Grundrechtsschutz seien zum Nulltarif ohne Übernahme eigener Verantwortung möglich, und legt diese voll in die Hände der Wirtschaft. Für Akzeptanz und wirksamen Grundrechtsschutz sind aber allgemein vertrauenswürdige Rahmenbedingungen nötig - nur auf dieser Basis kann sich das Geschäftsfeld Datenschutzzertifizierung für private Prüfgesellschaften erfolgreich entwickeln.

Die Erfahrungen des Unabhängigen Landeszentrums für Datenschutz (ULD) in seiner über 10-jährigen Zertifizierungspraxis zeigen, dass Transparenz und eine qualifizierte unabhän- gige Zertifizierungsstelle für vertrauenswürdige und erfolgreiche Verfahren unabdingbar sind. Dazu fehlen bei den Regierungsplanungen bisher alle Voraussetzungen: genügend technisch und rechtlich qualifiziertes Personal, die sinnvolle Einbindung der Expertise der Datenschutzaufsicht und schlanke, fachlich orientierte Verfahren. Geplant ist bisher nicht viel mehr als eine Geschäftsstelle, die vorrangig einen wirtschaftsgetriebenen Debattierclub koordiniert. Der aufgeblähte Beirat wird selbst vom ITBranchenverband BITKOM kritisiert. Spätestens seit der aktuellen Vorlage des Entwurfs der Europäischen Kommission für eine Datenschutz-Grundverordnung sollte auch der Bundesregierung klar sein, dass die Zukunft des Datenschutzes nicht in einem Schachern über Bedingungen der personenbezogenen Datenverarbeitung liegt, sondern in der präventiven Sicherung von „Compliance“ - also der Beachtung verpflichtender Datenschutznormen.

Es besteht die Gefahr, dass sich hier Deutschland mit einem kleinkarierten und bürokratischen Ansatz die Chance verbaut, europa- und weltweit in der aufstrebenden Datenschutzbranche führend zu sein. In Art. 39 des Entwurfs einer DatenschutzGrundverordnung ist die Förderung der „Einführung von datenschutzspezifischen Zertifizierungsverfahren sowie von Datenschutzsiegeln und -zeichen, anhand deren betroffene Personen rasch das von für die Verarbeitung Verantwortlichen oder von Auftragsdatenverarbeitern gewährleistete Datenschutzniveau in Erfahrung bringen können“, vorgesehen. Diese Steilvorlage könnte und sollte die Bundesregierung aufgreifen und das realistische Ziel verfolgen, in diesem Bereich die Vorreiterrolle Deutschlands bei der Datenschutzzertifizierung weiter auszubauen. Dafür darf man aber nicht kleckern, muss man klotzen. Die Erfahrungen und die Kooperationsbereitschaft der unabhängigen Datenschutzbehörden sind zu nutzen. Von all dem ist bei der „Stiftung Datenschutz" nichts zu erkennen. Wirklicher Grundrechtsschutz und effektive Wirtschaftsförderung gehen anders.

Dr. Thilo Weichert, Landesbeauftragter für Datenschutz des Landes Schleswig-Holstein und damit Leiter des ULD 